\title{
Cross-Correlations in Warsaw Stock Exchange
}

\author{
R. RAK ${ }^{a}$, J. KWAPIEŃ ${ }^{b}$, S. DROŻD $\dot{Z}^{a, b}$ AND P. OŚWIȨCIMKA ${ }^{b}$ \\ ${ }^{a}$ Institute of Physics, University of Rzeszów \\ Rejtana 16A, PL-35-959 Rzeszów, Poland \\ ${ }^{b}$ Institute of Nuclear Physics, Polish Academy of Sciences \\ PL-31-342 Kraków, Poland
}

\begin{abstract}
We study the inter-stock correlations for the largest companies listed on Warsaw Stock Exchange and included in the WIG20 index. Our results from the correlation matrix analysis indicate that the Polish stock market can be well described by a one-factor model. We also show that the stock- stock correlations tend to increase with the timescale of returns and they approach a saturation level for the timescales of at least $200 \mathrm{~min}$, i.e. an order of magnitude longer than in the case of some developed markets. We also show that the strength of correlations among the stocks crucially depends on their capitalization. These results combined with our earlier findings together suggest that now the Polish stock market situates itself somewhere between an emerging market phase and a mature market phase.
\end{abstract}

PACS numbers: 89.20.-a, 89.65.Gh, 89.75.-k

\section{Introduction}

Since the pioneering work of Markowitz in 1950s [1], the financial cross-correlations are constantly a subject of extensive studies both at the theoretical and practical levels due to their fundamental relation to risk management and portfolio investing. In the field of econophysics, an interest in this type of correlations arose after it had been shown that they can be described [2-4] in the framework of random matrix theory (RMT) [5], expressing both a kind of universality and significant deviations from it. Stock market cross-correlations are typically quantified in terms of a correlation matrix, created for a set of $N$ time series representing returns of different stocks. From this point of view, evolution of a stock market can be decomposed into $N$ independent modes associated with eigenvalues of the correlation matrix. It occurs that a vast majority of these eigenvalues are concordant with the eigenvalue distribution of the relevant random matrix ensemble (the Wishart ensemble) $[2,6]$, which according to a common belief suggests that 
these RMT modes do not carry any market-specific information beyond being a pure noise. Validity of this belief, however, has recently been challenged in some works [7]. As regards the remaining minority of the eigenvalues which deviate from the RMT predictions, there is a general agreement that they express the actual non-random linear dependences between the price fluctuations of different assets. Their particular number depends on a market and the number of analyzed stocks, but in each case there is an eigenvalue that strongly dominates, developing an "energy gap" that separates it from the subsequent eigenvalues. This peculiar eigenvalue is associated with a strongly delocalized eigenvector and is related to a "market mode", i.e. a collective evolution of large group of stocks that usually closely mimics evolution of the market's global index. From this perspective, magnitude of the largest eigenvalue reflects how collective is the evolution of an analyzed market. If, apart from the largest eigenvalue, there are also other eigenvalues which do not agree with the RMT spectrum, they correspond to smaller groups of interrelated stocks that can be usually identified with market sectors [4]. It has been found that the number of the non-random eigenvalues is highest for the largest, mature markets like New York, London, Frankfurt etc., while the less capitalized markets, e.g. the emerging ones, develop the spectrum which consists of a strongly repelled eigenvalue and the bulk with only minor disagreements in respect of the RMT prediction [8]. In fact, on small markets sectors and individual companies are too weak to be considered an optimal reference for the investors. Instead, the investors trade according to the behavior of the whole market or even they blindly follow the moves of the world's largest markets.

Here we analyze high-frequency data from the Warsaw Stock Exchange (WSE) and inspect the correlation matrix eigenspectra calculated for a few selected groups of stocks. We address the question whether the correlation properties of the Warsaw stock market still situate it among the emerging markets or, conversely, it has already matured enough to be considered a developed market. An inspiration for rising this question is the fact that the WSE evolution shares some properties (like the broad multifractal spectra and the returns distributions which can be fitted by the $q$-Gaussians) with the well-established markets, as our earlier studies showed [9-11].

\section{Methodology}

Our tick-by-tick data covered the period from 17 November 2000 to 30 June 2005 and consisted of 39 stocks that were, at least temporarily, included during this period in WIG20 index. WIG20 is a capitalization-weighted index comprising the 20 largest companies traded on WSE. Its composition changes from time to time in order to reflect the current capitalization ranking of the WSE stocks. During the forementioned time interval only 10 stocks were constantly included in WIG20, while each of the remaining 29 stocks was contributing to WIG20 for a shorter period due to falling in the capitalization ranking or being delisted from WSE. 


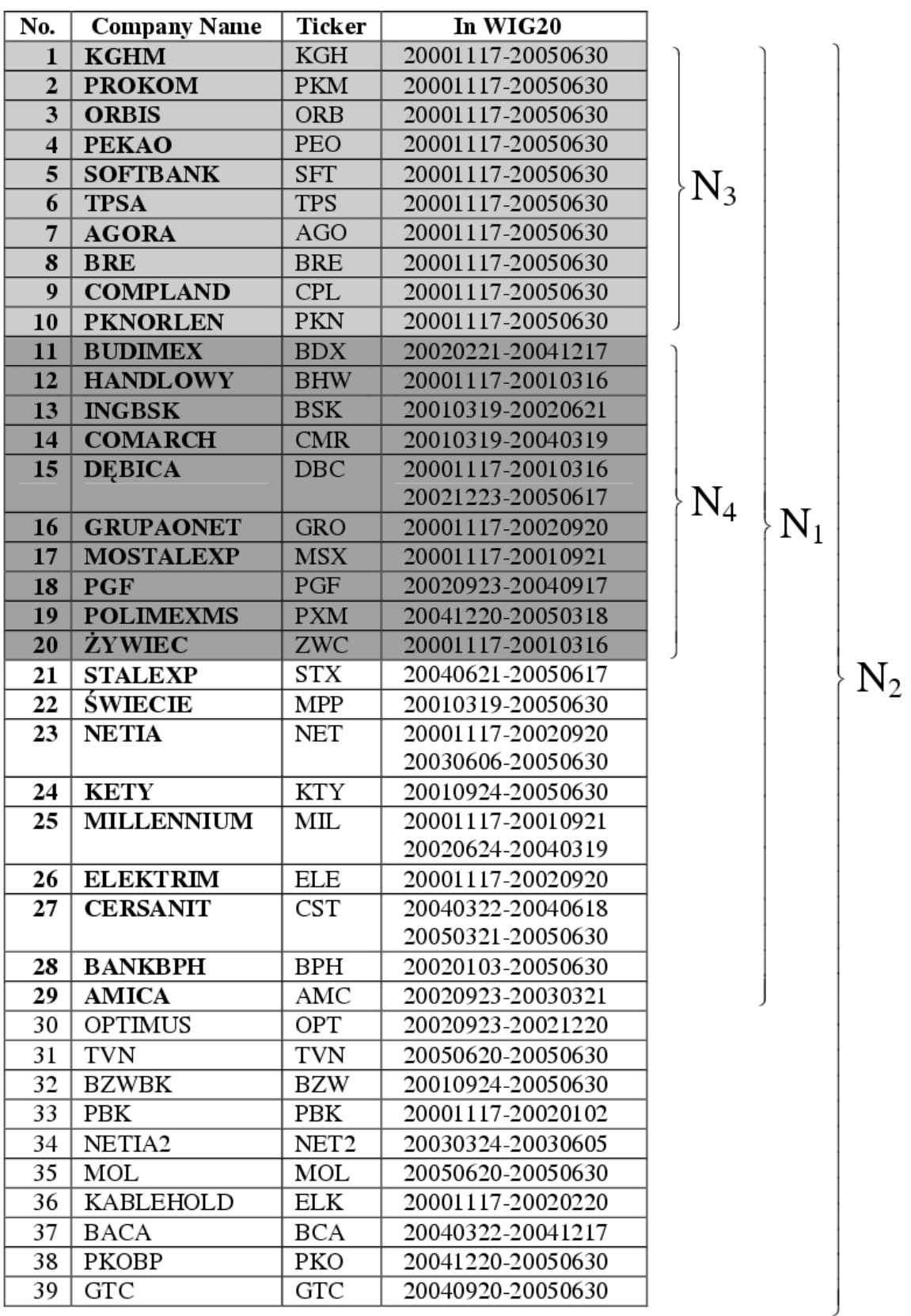

Fig. 1. Stocks included in WIG20 during the period 17.11.2000-30.06.2005, divided into four groups $N_{1}, N_{2}, N_{3}, N_{4}$. See text for details.

Although it is associated with a relatively small number of companies, WIG20 is considered the most important and influential index on WSE. This 
is because the companies included in WIG20 are viewed as belonging to a core of Polish economy. Thus it is not unreasonable to expect that their statistical and correlation properties differ, at least to some extent, from the properties of other companies that are not a part of this index. Also a particular stock may change its behavior after being included in or removed from WIG20. In order to investigate this issue we divided our set of signals into 4 partially overlapping groups (see Fig. 1):

(1) Group 1 of $N_{1}=29$ signals associated with the stocks listed on WSE over the entire period.

(2) Group 2 of $N_{2}=20$ signals constituting WIG20 with changing stock content according to the actual WIG20 basket composition; signals (at the level of normalized returns) representing the replaced stocks were cut and joined with the ones representing the replacing stocks.

(3) Group 3 of $N_{3}=10$ signals for the stocks that were permanently included in WIG20; these are also the companies with the largest capitalization.

(4) Group 4 of $N_{4}=10$ signals representing the stocks with the least capitalization among the ones that were temporarily included in WIG20. We performed our calculations for each of the above groups independently.

For each individual company $\alpha, \alpha=1, \ldots, N_{k}$, from the raw tick-by-tick data we extracted a time series of price evolution $p_{\alpha}\left(t_{i}\right), i=1, \ldots, T$ sampled with $1 \mathrm{~min}$ frequency and calculated the corresponding returns according to the usual definition: $G_{\alpha}\left(t_{i}\right)=\ln p_{\alpha}\left(t_{i}+\tau\right)-\ln p_{\alpha}\left(t_{i}\right)$, where $\tau$ is the time lag. After normalizing the time series of returns to have unit variance and zero mean, the resulting length of each signal was equal to $T=415,000$.

From $N_{k}$ time series we construct an $N_{k} \times T$ data matrix $\boldsymbol{M}$ and the correlation matrix $C$ that are related by

$$
\boldsymbol{C}=(1 / T) \boldsymbol{M} \boldsymbol{M}^{\mathrm{T}} .
$$

By diagonalizing the correlation matrix

$$
\boldsymbol{C} \boldsymbol{v}^{j}=\lambda_{j} \boldsymbol{v}^{j}
$$

one obtains a set of its eigenvalues $\lambda_{j}, j=1, \ldots, N_{k}$ and eigenvectors $\boldsymbol{v}^{j}=\left\{v_{\alpha}^{j}\right\}$.

An ensemble of random matrices which can be used as a null hypothesis in our context is the ensemble of the Wishart matrices. It offers an analytic expression for a distribution of eigenvalues, known as the Marchenko-Pastur formula [12, 6]. Here we are interested in its upper $\lambda_{\max }$ and lower $\lambda_{\min }$ bounds only

$$
\lambda_{\min }^{\max }=\sigma^{2}(1+1 / Q \pm 2 \sqrt{1 / Q}),
$$

with $Q=T / N_{k} \geq 1$ and time series variance $\sigma^{2}=1$.

\section{Results}

Figure 2 shows the eigenvalue spectra for each of the 4 considered groups of stocks; two time lags are used: $\tau=10 \mathrm{~min}$ (top) and $\tau=360 \mathrm{~min}$, i.e. 1 trading day (bottom). In accordance with the remarks done in the introductory section, for 

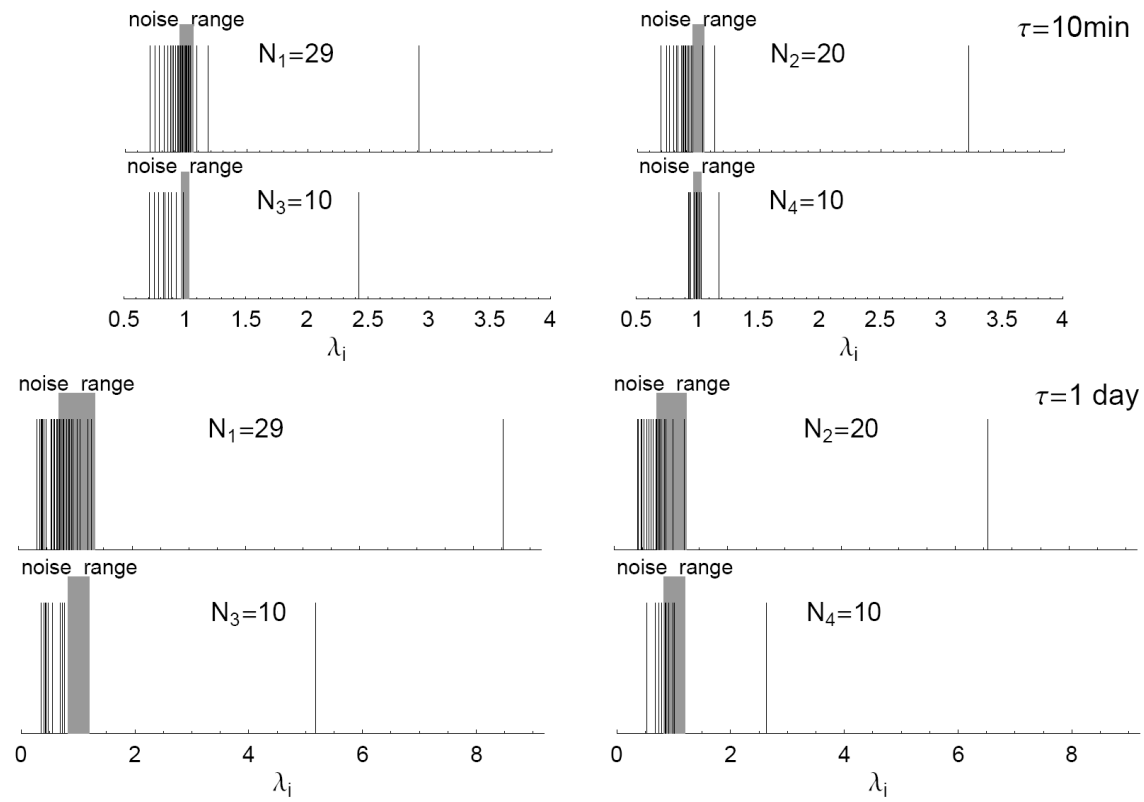

Fig. 2. Empirical eigenvalue spectrum of the correlation matrix $\boldsymbol{C}$ (vertical lines), calculated for 4 group of companies over the period 17.11.2000-30.06.2005. Two timescales are shown: $\tau=10 \mathrm{~min}$ (top) and $\tau=360 \mathrm{~min}=1$ day (bottom). According to RMT predictions, eigenvalues of a Wishart matrix have to lie only within the shaded region.

all the groups and for both time lags the largest eigenvalue $\lambda_{1}$ is repelled from the RMT range defined by Eq. (3). Clearly, for Groups 1, 2, 3 the corresponding shift is stronger than for Group 4. As regards the rest of the eigenvalues, they are close to the random matrix region; the observed discrepancies between their position and the RMT bounds can be attributed to the "squeezing" effect of large $\lambda_{1}$ exerted on the smaller eigenvalues $[2,7]$ which are shifted towards zero. The larger magnitude of $\lambda_{1}$, the stronger is this effect. Agreement between the empirical eigenspectrum and the RMT prediction can be significantly improved by removing a mode associated with $\lambda_{1}$ from the analyzed signals (see e.g. Ref. [7, 13]). The largest eigenvalue is related to the temporal evolution of the market mode. As we see in Fig. 2, practically no other eigenvalue considerably exceeds the RMT upper bound $\lambda_{\max }$, which indicates that — besides the idiosyncratic fluctuations the market mode is the principal and unique factor responsible for the behavior of individual stocks. A lack of other deviating eigenvalues is the evidence of weakness of a sectorization in the WSE market.

The magnitudes of the largest eigenvalues for different groups of stocks cannot be directly compared based on Fig. 2. This is because of a lack of a proper normalization: the correlation matrices for different groups have different size and different trace. Thus, in each case we divide $\lambda_{1}$ by the matrix trace. As a result 
we obtain normalized values of $\lambda_{1}$ expressing a fraction of the maximum possible magnitude (equal to the matrix trace) that is absorbed by the largest eigenvalue. It thus describes the "rigidity" of a given group's temporal evolution. The corresponding results are exhibited in Fig. 3. For both timescales, the largest normalized magnitude of $\lambda_{1}$ is observed for Group 3, comprising the largest companies permanently listed in WIG20. The difference between this and other groups is especially substantial for the larger timescale of 1 trading day (Fig. 3, bottom part). This indicates that the stocks for the largest companies are particularly strongly coupled with each other. Evidently smaller strength of collective movements can be seen for Group 2 (representing the actual content of WIG20), which is associated with the second-largest magnitude of $\lambda_{1}$. The stocks within the remaining Groups 3 and 4 are relatively weakly correlated. In contrast to Groups 1 and 2, which at each moment comprise the stocks belonging to the WIG20 basket, the stocks from Groups 3 and 4 are not necessarily included in WIG20 during the whole studied interval of time: at each particular moment some of them belong to WIG20 and some of them do not. This also means that their capitalization is, on average, smaller than the one for the stocks from Groups 1 and 2.

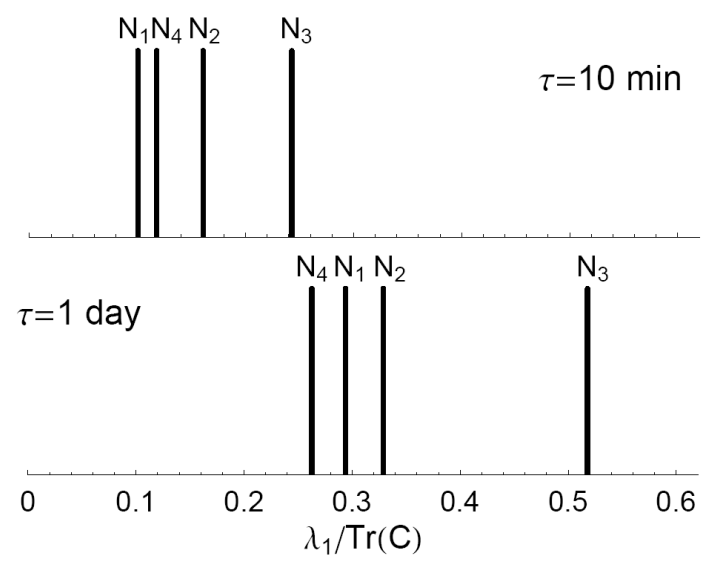

Fig. 3. Empirical eigenvalue spectrum of the correlation matrix $C$ (vertical lines), calculated for 4 groups of companies over the period 17.11.2000-30.06.2005. Two timescales are presented: $\tau=10 \mathrm{~min}$ (top) and $\tau=360 \mathrm{~min}=1$ day (bottom).

By comparing the results in both parts of Fig. 3, one can notice that for the longer timescale the eigenvalues assume larger magnitudes than do their counterparts for the shorter timescale. This can be a manifestation of the Epps effect $[14,15]$, i.e. increase in market cross-correlations with increasing timescale of the returns. In order to verify this supposition, we systematically inspected the functional dependence of $\lambda_{1}$ on time lag $\tau$ for a few distinct timescales $10 \mathrm{~min}$ $<\tau<900$ min. Indeed, the results collected in Fig. 4 confirm that the observed behavior of the largest eigenvalue is a consequence of the Epps effect. For all the 


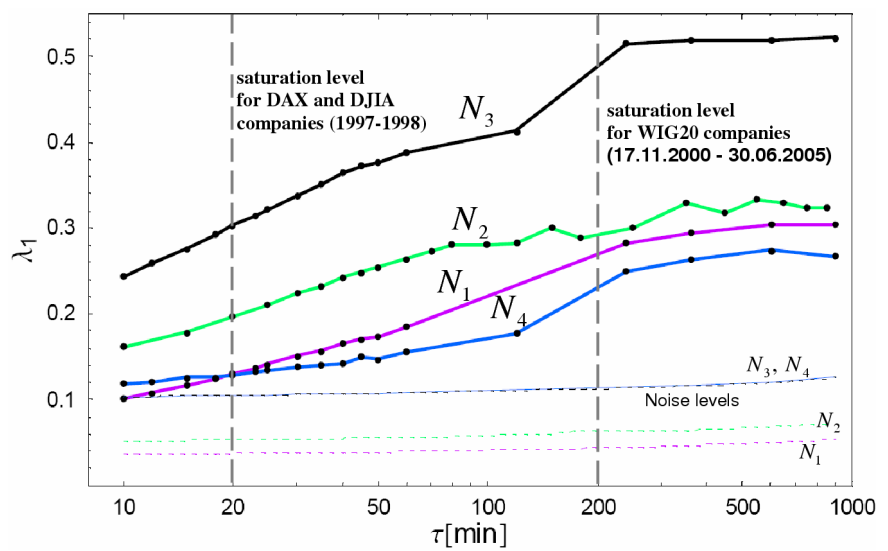

Fig. 4. Functional dependence of $\lambda_{1}$ on time lag $\tau$ for all analyzed groups of stocks.

groups, $\lambda_{1}$ increases from small values for the shortest time lags to the group-specific saturation levels for $\tau>200 \mathrm{~min}$ (compare with the American market with similar saturation occurring for $\tau>20 \mathrm{~min}$ ). It is noteworthy that for Group 3 and Group 4 and for $\tau=10$ min the largest eigenvalue is comparable in size with the noise level $\left(\lambda_{\max }\right)$ predicted by RMT. This outcome resembles the analogous one obtained for the American stock market [15]: the smaller is the capitalization of a group of stocks, the less internally correlated and more noisy is the group's evolution, which — in consequence with the Epps effect — leads to a complete lack of actual inter-stock couplings for sufficiently short timescales. This phenomenon is related to the fact that investors need some time to fully react to new information and events on a stock market. Stocks of smaller companies are traded less frequently than stocks of large companies and therefore the amount of time needed to develop couplings between such stocks is considerably larger.

\section{Summary}

We investigated the inter-stock correlations for the relatively large companies traded on Warsaw Stock Exchange and included in the WIG20 index. We divided the full set of stocks into 4 groups depending on a particular stock's capitalization and a time interval in which the stock was included in WIG20. Our results show that the Polish stock market can basically be expressed by a one-factor model with the fully developed couplings to occur at timescales longer than half a trading day. Since these properties are characteristic of small and emerging markets and since, on the other hand, the Polish market reveals some features that are common to well-developed markets ( $q$-Gaussian structure of the returns probability density functions (p.d.f.s) [10], multifractality [11]), we arrive at the conclusion that at present WSE is in a transition phase from being an emerging market to becoming a fully-established one. Our analysis also proved that the 
strength of correlations among stocks crucially depends on their capitalization this effect is universal for all the markets investigated so far in literature.

\section{References}

[1] H. Markowitz, J. Finance 7, 77 (1952).

[2] L. Laloux, P. Cizeau, J.-P. Bouchaud, M. Potters, Phys. Rev. Lett. 83, 1467 (1999).

[3] V. Plerou, P. Gopikrishnan, B. Rosenow, L.A.N. Amaral, H.E. Stanley, Phys. Rev. Lett. 83, 1471 (1999).

[4] V. Plerou, P. Gopikrishnan, B. Rosenow, L.A.N. Amaral, T. Guhr, H.E. Stanley, Phys. Rev. E 65, 066126 (2002).

[5] M.L. Mehta, Random Matrices, Academic Press, Boston 1999.

[6] A.M. Sengupta, P.P. Mitra, Phys. Rev. E 60, 3389 (1999).

[7] J. Kwapień, S. Drożdż, P. Oświęcimka, Physica A 359, 589 (2006).

[8] R.K. Pan, S. Sinha, arxiv.org/physics/0704.0773 (2007).

[9] P. Oświęcimka, J. Kwapień, S. Drożdż, A.Z. Górski, R. Rak, Acta Phys. Pol. B 37, 3083 (2006).

[10] R. Rak, S. Drożdż, J. Kwapień, Physica A 374, 315 (2007).

[11] S. Drożdż, M. Forczek, J. Kwapień, P. Oświęcimka, R. Rak, Physica A 383, 59 (2007).

[12] V.A. Marchenko, L.A. Pastur, Math. USSR-Sbornik 1, 457 (1967).

[13] S. Drożdż, J. Kwapień, P. Oświęcimka, Acta Phys. Pol. B 13, 4027 (2007).

[14] T.W. Epps, J. Am. Stat. Assoc. 74, 291 (1979).

[15] J. Kwapień, S. Drożdż, J. Speth, Physica A 337, 231 (2004). 